\title{
Ultrafast Nanomagnetic Toggle Switching of Vortex Cores
}

\author{
R. Hertel, ${ }^{1, *}$ S. Gliga, ${ }^{1}$ M. Fähnle, ${ }^{2}$ and C. M. Schneider ${ }^{1}$ \\ ${ }^{1}$ Institut für Festkörperforschung IFF-9 “Elektronische Eigenschaften,” Forschungszentrum Jülich GmbH, D-52425 Jülich, Germany \\ ${ }^{2}$ Max-Planck-Institut für Metallforschung, D-70569 Stuttgart, Germany
}

(Received 10 October 2006; published 12 March 2007)

\begin{abstract}
We present an ultrafast route for a controlled, toggle switching of magnetic vortex cores with ultrashort unipolar magnetic field pulses. The switching process is found to be largely insensitive to extrinsic parameters, like sample size and shape, and it is faster than any field-driven magnetization reversal process previously known from micromagnetic theory. Micromagnetic simulations demonstrate that the vortex core reversal is mediated by a rapid sequence of vortex-antivortex pair creation and annihilation subprocesses. Specific combinations of field-pulse strength and duration are required to obtain a controlled vortex core reversal. The operational range of this reversal mechanism is summarized in a switching diagram for a $200 \mathrm{~nm}$ Permalloy disk.
\end{abstract}

PACS numbers: $75.40 . \mathrm{Gb}, 75.40 . \mathrm{Mg}, 75.60 . J k, 75.75 .+\mathrm{a}$

Ferromagnetic materials in confined geometries typically form domain structures that close the magnetic flux [1]. In the center of such flux-closure structures there is a region of only a few nanometers in size known as a magnetic vortex, where the magnetization circulates around a core [2-4]. In the vortex core, the magnetization points out of the vortex plane, thereby preventing a singularity of the exchange energy density. Owing to the dramatic improvement in the spatial resolution of magnetic imaging techniques, it has recently become possible to directly observe the nanometric region of the vortex core [3] and to study vortex dynamics [5-8].

In analytical models for vortex dynamics [9], the core is usually assumed to be a rigid magnetic structure that remains unchanged when its position is shifted, e.g., under the influence of an external field. The high structural stability of vortex cores results from the strong exchange interaction. Nevertheless, recent studies have shown that the internal magnetic structure of a vortex core can be modified temporarily by magnetic fields applied in the film plane [10-12]. Short of its annihilation with an antivortex [13], the most drastic possible modification of a magnetic vortex structure is the reversal of its perpendicular core, i.e., the switching of the vortex polarization.

A field-driven switching of the vortex polarization has been shown to be possible by applying a static magnetic field $[14,15]$ perpendicular to the vortex plane, thus "crushing" the vortex core and reestablishing it in the opposite direction. This process requires field strengths of the order of $500 \mathrm{mT}$. Such a large field value indicates that a high energy barrier must be surmounted to switch a magnetic vortex core. This high barrier ensures high thermal stability, which in combination with their well-defined orientation and their extremely small size makes vortex cores interesting candidates for binary data storage [16]. The first demonstration that vortex cores can also be switched by low-amplitude in-plane magnetic fields [17] has been provided only very recently [8]. In the experiment of Ref. [8], short oscillating magnetic field pulses of low amplitude were used, tuned to the gyrotropic resonance frequency of the system [5]. The gyrotropic frequency depends on the particle size and shape and is typically in the order of a few $100 \mathrm{MHz}$. A weak oscillating resonant magnetic field induces a rotation of the vortex on a stationary orbit. Exploiting the sense of rotation, it was demonstrated that the vortex core reversal can be triggered with weak sinusoidal field pulses of about 4 ns duration and strength of about $1.5 \mathrm{mT}$ [8].

We have studied the dynamics of vortex core reversal with micromagnetic simulations using a fully threedimensional finite-element algorithm based on the Landau-Lifshitz-Gilbert equation [18]. Our simulations show that the time scale required for a vortex core reversal is not limited by the relatively slow gyrotropic resonance frequency: A vortex core reversal process can also be triggered by a nonresonant, unipolar, and very short field pulse (below $100 \mathrm{ps})$ of moderate strength $(\sim 80 \mathrm{mT})$ applied parallel to the film plane of, e.g., a submicron sized $\mathrm{Ni}_{81} \mathrm{Fe}_{19}$ (Permalloy, Py) disk (Fig. 1). In this study we also provide a detailed description of the transformation undergone by the vortex during this process: The core reversal occurs through a sequence consisting of a vortexantivortex pair creation, followed by an annihilation pro-

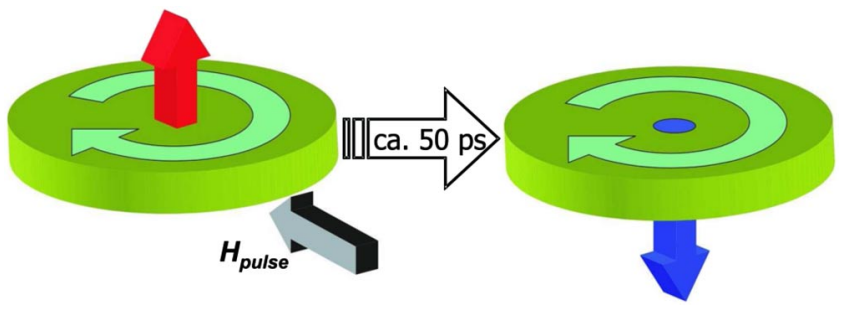

FIG. 1 (color online). Schematics of a field-pulse driven vortex core switching. The vortex core magnetization can be switched by a short magnetic field pulse applied in the film plane. This switching process requires only $40-50 \mathrm{ps}$. 
cess, resulting in a final magnetic structure of a single vortex with opposite polarization, in agreement with the model suggested in Ref. [8]. We find that the time required for the core reversal is of the order of $40 \mathrm{ps}$ [19]. This is a very high speed for a reversal process, which is, e.g., about 5 times faster than that involved in ultrafast precessional switching mechanisms [20].

A typical example of the vortex-antivortex pair creation mediated core switch process is shown in Fig. 2. For the simulation of this Py disk (radius of $100 \mathrm{~nm}$ and thickness of $20 \mathrm{~nm}$ ) we have used about 150000 tetrahedral elements, corresponding to a cell size of $3 \mathrm{~nm}$. The material parameters chosen for Py are the following: $A=13 \mathrm{pJ} / \mathrm{m}$ (exchange constant), $\mu_{0} M_{s}=1.0 \mathrm{~T}\left(M_{s}\right.$ : saturation magnetization), and $K_{u}=0$ ( $K_{u}$ : magnetocrystalline anisotropy), corresponding to the usual room-temperature values [21]. The Gilbert damping constant was set to $\alpha=$ 0.01. An $80 \mathrm{mT}$ Gaussian shaped field pulse of a duration of $60 \mathrm{ps}$ is applied in the plane of the Py disk, which is initially in a symmetric vortex state. The microscopic processes leading to the core reversal can be clearly identified by highlighting the $m_{x}=0$ and $m_{y}=0$ isosurfaces [13], the intersection of which determines the exact position of the vortex core. Before an external field is applied, these $m_{x}=M_{x} / M_{s}=0$ and $m_{y}=M_{y} / M_{s}=0$ isosurfaces appear as straight ribbons (oriented parallel to the $x$ and $y$ axis, respectively) crossing each other perpendicularly at the center of the vortex core. As the field pulse perturbs the system, the vortex shifts away from its original position and the formerly circular arrangement of the magnetization around the core is stretched in one direction, resulting in bent isosurfaces. A few picoseconds after the peak value of the pulse is reached, the isosurfaces are bent strongly enough to form two additional intersections. These intersections mark the creation of a vortex-antivortex pair [13]. The magnetization direction in the core of the new vortex is opposite to the polarization of the original core.

Once a pair is created, the antivortex quickly moves towards the original vortex and, through a rapid process, they annihilate each other. The details of the magnetization dynamics of such annihilation processes, which include the formation and the propagation of a micromagnetic singularity (Bloch point), have been reported in Ref. [13]. It has been shown that this vortex-antivortex annihilation is connected with a sudden generation of spin waves [22]. After the sequence of pair creation and annihilation processes, the magnetic structure is again in a vortex state, but with opposite polarization with respect to the original vortex. The core switching does not affect the in-plane sense of rotation of the magnetization, hence resulting in a change of the vortex handedness [5].

The change of the perpendicular core magnetization, while not explicitly shown in Fig. 2, can be seen in Fig. 3. The reversed core magnetization is clearly obtained by the creation of a new oppositely magnetized area, which evolves into a vortex-antivortex structure. Subsequently, the original core annihilates with the new antivortex. The starting point for the formation of the out-of-plane compo-

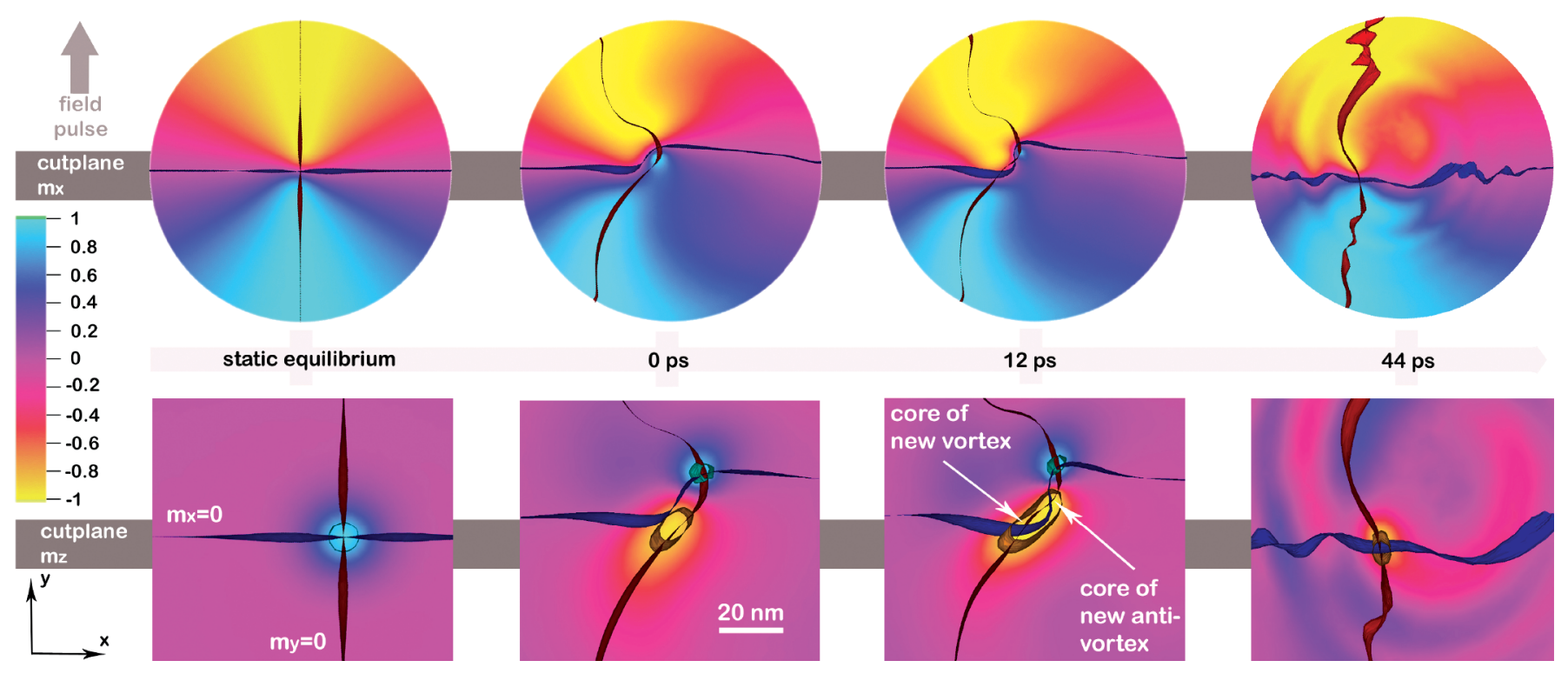

FIG. 2 (color). Pair-creation mediated vortex core reversal in a Py disk of $100 \mathrm{~nm}$ radius and $20 \mathrm{~nm}$ thickness. A Gaussian field pulse is applied in the disk plane, parallel to the $y$ axis. The top row shows the $x$ component of the magnetization $m_{x}$ in the initial state, followed by the state of the sample at the pulse maximum (" $0 \mathrm{ps")}$ ) and then at two different times after the pulse maximum has been reached. The blue and red ribbons represent the $m_{x}=0$ and $m_{y}=0$ isosurfaces, respectively. The bottom row shows a magnification of the region where these ribbons intersect, marking the cores of the original vortex, then at 12 ps after the pulse maximum, of the newly created vortex-antivortex pair, and finally of the remaining vortex core. This core has opposite polarization with respect to the initial vortex core, indicated by the yellow color on the underlying cut plane representing the $z$ component of the magnetization $m_{z}$. The green and orange cylindrical ribbons are the isosurfaces where $m_{z}=0.8$ and $m_{z}=-0.8$, respectively. 

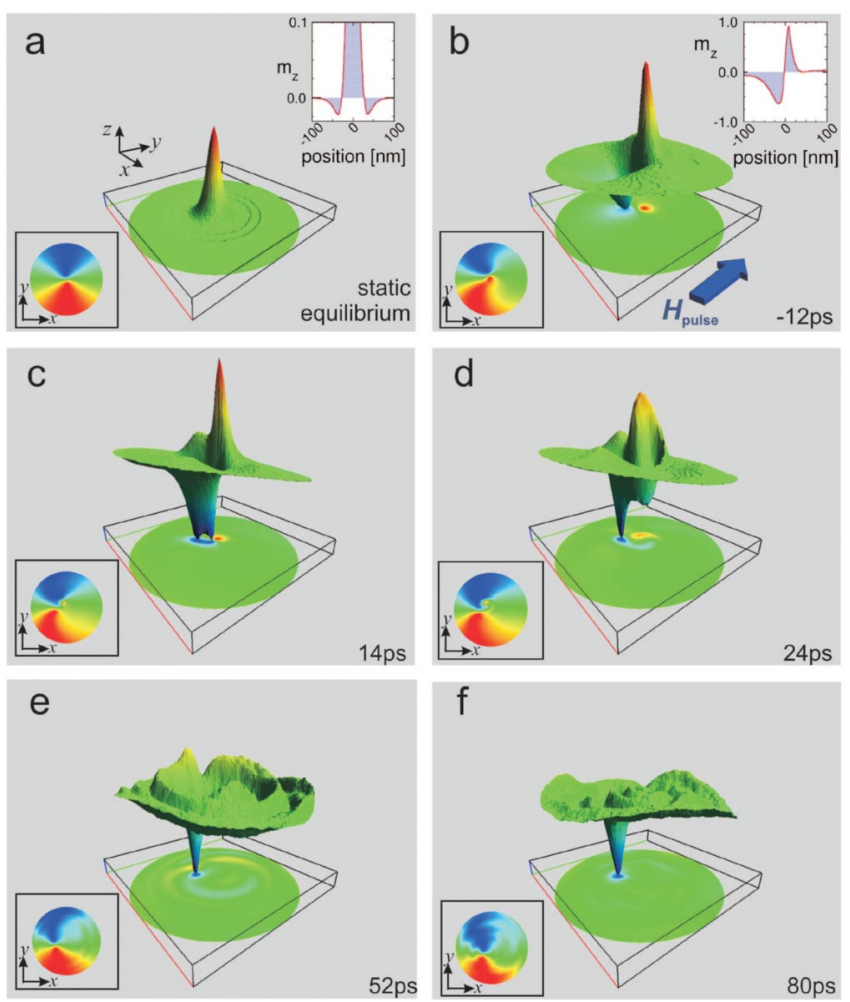

FIG. 3 (color). Topography of $m_{z}$ during the core switching process. (a) Static equilibrium. The cutline on the upper right shows the small negative dip in $m_{z}$ near the core. (b) Formation of a large negative dip of $m_{z}$. The cutline goes along the diameter through the vortex core and the adjacent dip. (c) Pair creation leads to two separate points with $m_{z}=-1$. (d) The original vortex core annihilates with the new antivortex. (e) Spin waves are emitted after the annihilation. Finally, a vortex core with opposite polarization remains (f). The insets on the lower left show the $x$ component of the magnetization (red: $m_{x}=1$, blue: $\left.m_{x}=-1\right)$. The times are relative to the pulse maximum.

nent of the new vortex is a "dip" in the perpendicular component of the magnetization, $m_{z}$, that forms close to the vortex core $[10,23]$ as the vortex is distorted; cf. Fig. 3(b). The formation of this out-of-plane component is due to the tendency of the system to reduce the exchange energy connected with the strong inhomogeneity of the inplane magnetic structure: as the $m_{x}=0$ and the $m_{y}=0$ isosurfaces approach each other, the distance over which the magnetization changes its in-plane direction by $90^{\circ}$ is reduced to only a few nanometers. The system circumvents the formation of such a strongly inhomogeneous structure by rotating the magnetization out of the plane. While the exchange field is responsible for the formation of a pronounced out-of-plane component in a distorted vortex structure, it is the magnetostatic field of the vortex core that is decisive for the direction into which this out-ofplane dip develops. In the close vicinity of the vortex core, there is a strong dipolar field originating from the core. This field provides a bias into the opposite direction of the vortex core magnetization. As shown on the cutline in Fig. 3(a), the dipolar field of the core affects the magnetic structure on a ring around the vortex core, leading to a negative "halo" of the $m_{z}$ component around the core [24]. The simulations yield that the vortex core gives rise to a negative perpendicular field component of about $200 \mathrm{mT}$ in the region where the dip develops [25].

The simulations further show that the pair-creation mediated vortex core reversal mechanism is insensitive to variations in particle shape or size. The same reversal process occurs as well in elliptical and square submicron sized magnetic thin-film elements. The process also does not seem to depend on the value of the damping coefficient $\alpha$ used in the Landau-Lifshitz-Gilbert equation [26]. Only the characteristics of the required field pulse depend on the individual sample properties. These results suggest that the field-pulse induced generation and the subsequent annihilation of magnetic vortex-antivortex pairs is a general property of magnetic vortices. In view of this general nature of the process, we assume that the pair-creation mediated core reversal could also be initiated by electric current pulses [23] or by laser pulses [27].

We found that the field-pulse induced core switch in our disk-shaped sample occurs for well-defined combinations of the applied pulse's duration and strength. The influence of these two parameters is shown in Fig. 4 for a $20 \mathrm{~nm}$ thick, $200 \mathrm{~nm}$ large Py nanodisk. This diagram was obtained by varying the pulse width $\sigma$ in steps of $2.5 \mathrm{ps}$ for $t=0 \mathrm{ps}$ to $t=20 \mathrm{ps}$ and in steps of $10 \mathrm{ps}$ thereafter. The pulse maximum was varied in increments of $5 \mathrm{mT}$. The precise position of the boundaries in the diagram is difficult to determine because they (weakly) depend on the size of the discretization cells. These variations in the critical field result from the mesh dependence of the Bloch point nucleation [15]. The operating field range is relatively narrow: While too low fields do not cause the core to switch, too high fields give rise to sequences of multiple pair

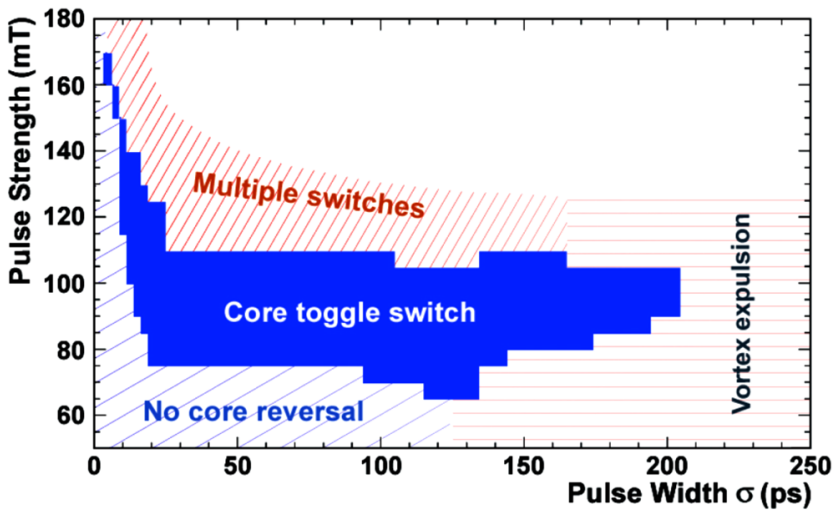

FIG. 4 (color online). Switching diagram for the pulse parameters leading to a toggle switch of the vortex core in a $100 \mathrm{~nm}$ radius and $20 \mathrm{~nm}$ thick Py disk. The pulse duration is quantified by its width $\sigma$ and the strength by its maximum value. The cell size was $3 \mathrm{~nm}$. It was varied by $\pm 0.7 \mathrm{~nm}$ at $80 \mathrm{mT} / 20 \mathrm{ps}$ (diagram edge), giving a variation in the field required to switch the core of $+5.0 \mathrm{mT}$ for $2.3 \mathrm{~nm}$ cells and $-5.6 \mathrm{mT}$ for $3.7 \mathrm{~nm}$ cells. 
creations and annihilations. It is observed that by extending the pulse duration, switching can be accomplished with pulses of $65 \mathrm{mT}$, while by increasing the applied field strength, it is possible to obtain switching with pulses only $5 \mathrm{ps}$ long. Ultimately, too strong and too long pulses lead to a temporary expulsion of the vortex from the sample.

In conclusion, we presented a detailed description of an ultrafast process in nanomagnetism for switching the core of magnetic vortices. Although this reversal mode involves a series of complex processes on the nanometer scale - the creation of a vortex-antivortex pair, a subsequent annihilation process - the chain of events only requires a short field pulse of suitable shape to be initiated. The ultrafast speed of this process is perhaps equally astounding as the finding that such a long sequence of subprocesses develops almost automatically.

The pair-creation mediated vortex core reversal is comparable in scope with the precessional switching mechanism [20]. However, the pair-creation and annihilation processes are driven by the exchange field, while the precessional switching exploits the demagnetizing field. The magnitude of the exchange field is in the order of $100 \mathrm{~T}$, which is about 100 times larger than the demagnetizing field. This explains the considerably higher speed of the core reversal, which is almost an order of magnitude faster. A further advantage of the vortex core reversal mechanism lies in the simplicity of the required sample and magnetic structure: all that is needed is a magnetic vortex, which is a structure that forms naturally in submicron sized magnetic disks [28]. The core reversal process described here therefore represents a significant leap towards smaller length scales and shorter time scales.

We thank H. Stoll (MPI Stuttgart) and co-workers for bringing the vortex switching problem to our attention.

Note added in proof.-After submission of this Letter, similar results were reported by Xiao et al. [29].

*Electronic address: r.hertel@fz-juelich.de

[1] A. Hubert and R. Schäfer, Magnetic Domains-The Analysis of Magnetic Microstructures (Springer, Berlin, New York, Heidelberg, 1998).

[2] T. Shinjo et al., Science 289, 930 (2000).

[3] A. Wachowiak et al., Science 298, 577 (2002).

[4] J. Miltat and A. Thiaville, Science 298, 555 (2002).
[5] S. B. Choe et al., Science 304, 420 (2004).

[6] J. P. Park et al., Phys. Rev. B 67, 020403(R) (2003).

[7] A. Puzic et al., J. Appl. Phys. 97, 10 E704 (2005).

[8] B. Van Waeyenberge et al., Nature (London) 444, 461 (2006).

[9] N. A. Usov and L. G. Kurkina, J. Magn. Magn. Mater. 242-345, 1005 (2002).

[10] V. Novosad et al., Phys. Rev. B 72, 024455 (2005).

[11] A. Neudert et al., J. Appl. Phys. 97, 10E701 (2005).

[12] K. S. Buchanan et al., Phys. Rev. B 74, 064404 (2006).

[13] R. Hertel and C. M. Schneider, Phys. Rev. Lett. 97, 177202 (2006).

[14] T. Okuno et al., J. Magn. Magn. Mater. 240, 1 (2002).

[15] A. Thiaville et al., Phys. Rev. B 67, 094410 (2003).

[16] R. Hollinger, A. Killinger, and U. Krey, J. Magn. Magn. Mater. 261, 178 (2003).

[17] J. P. Zagorodny et al., Eur. Phys. J. B 31, 471 (2003).

[18] R. Hertel, W. Wulfhekel, and J. Kirschner, Phys. Rev. Lett. 93, 257202 (2004).

[19] The time required for pair creation depends on the pulse profile. However, the subsequent annihilation seems to occur within a constant time of about 20 ps.

[20] T. Gerrits et al., Nature (London) 418, 509 (2002).

[21] Thermal fluctuations have not been considered explicitly in this study.

[22] K.-S. Lee, S. Choi, and S.-K. Kim, Appl. Phys. Lett. 87, 192502 (2005).

[23] S. Kasai et al., Phys. Rev. Lett. 97, 107204 (2006).

[24] J. K. Ha, R. Hertel, and J. Kirschner, Phys. Rev. B 67, 064418 (2003).

[25] The importance of the dipolar field in selecting the polarization of the vortex-antivortex pair has been shown by applying various out-of-plane field pulses parallel to the original vortex core polarization in addition to an in-plane pulse. The pair-creation process that is usually induced by the in-plane pulse is suppressed if the out-of plane pulse exceeds a magnitude of about $200 \mathrm{mT}$, corresponding to the value of the dipolar field strength.

[26] See EPAPS Document No. E-PRLTAO-98-083710 for three movies: movie1.avi details the isosurface representation; movie2.avi shows the vortex core reversal in the disk sample presented in Fig. 2; and movie3.avi shows the same process observed in a $300 \times 125 \times 20 \mathrm{~nm}$ Py elliptical element where $\alpha=0.5$. The visualization was done using GMV: http://www-xdiv.lanl.gov/XCM/GMVHome. html. For more information on EPAPS, see http://www. aip.org/pubservs/epaps.html.

[27] A. V. Kimel et al., Nature (London) 435, 655 (2005).

[28] R. P. Cowburn et al., Phys. Rev. Lett. 83, 1042 (1999).

[29] Q.F. Xiao et al., Appl. Phys. Lett. 89, 262507 (2006). 